\title{
The Astrometric Study of ADS 48 - a Nearby Double Star with a Probable Invisible Satellite ${ }^{1}$
}

\author{
O. V. KIYAEVA \\ Pulkovo Observatory, St. Petersburg, Russia
}

\begin{abstract}
The orbit of the visual double star ADS $48 \mathrm{AB}$ is determined on the basis of Pulkovo and Washington sets of observations. The similar deviations in observations of the both sets are discovered. It may be explained by the existence of the invisible satellite with a mass about $0.01 \mathcal{M}_{\odot}$.
\end{abstract}

The dynamical method of apparent motion parameters (AMP) was proposed and investigated at the Pulkovo Observatory for determining the orbit elements of a visual double star with a long period, similar to the nearby red dwarf binary ADS $48 \mathrm{AB}$.

This visual double star is observed from 1877 , and it was repeatedly investigated by different authors. When we investigated the AMP-method, this star was a prototype for a lot of model examples, its AMP-orbit was calculated on the basis of the short-arc photographic observations made in Pulkovo with the 26 -inch refractor from 1960 til 1985 (Kisselev \& Kiyaeva 1988). Our orbit was in agreement with ancient observations and coincided with a Güntzel-Lingner (1955) one within the error box. However, the sum of mass of the components $\mathcal{M}_{A+B}=2.0 \mathcal{M}_{\odot} \quad\left(\mathrm{a}=6^{\prime \prime}{ }^{\prime} 1, \mathrm{P}=370\right.$ years $)$ was more than twice as much as the value $\mathcal{M}_{A+B}=0.9 \mathcal{M}_{\odot}$ corresponding to the "mass-luminosity" relation.

To find out the reason, we decided to repeat this work after carrying out a more thorough analysis of the original data. All 113 plates observed from 1968 til 1989 were measured once more with the automatic measuring instrument "Parsec". All observations were made with the same kind of plates W0-1, the exposure time was 1 minute. Thus we provided one of the main conditions for using the AMP-method - a uniformity of the observational data.

As a result of a mathematical reduction by the least-squares method, there were obtained the following values for the unit-weight errors of the one plate:

$$
\sigma_{1 \rho}=0^{\prime \prime} .019 \quad \sigma_{1 \theta}=0.13 \quad \sigma_{1 \tau}=\rho \sigma_{1 \theta} / 57.3=0^{\prime \prime} .014
$$

The similar errors of the one normal place (mean within the year) are as follows:

$$
\sigma_{1 \rho}=0^{\prime \prime} .009 \quad \sigma_{1 \theta}=0^{\circ} .076 \quad \sigma_{1 \tau}=0^{\prime \prime} .008
$$

We solved for the following five apparent motion parameters at the moment $t_{0}$, corresponding to the middle of a set of short arc observations:

$$
\begin{aligned}
\rho, \theta & =\text { polar coordinates of the component } \mathrm{B} \text { relative to } \mathrm{A} ; \\
\mu & =\text { the angular velocity of the apparent motion } \mathrm{B} \text { relative to } \mathrm{A} ; \\
\Psi & =\text { the position angle of the apparent motion direction; }
\end{aligned}
$$

\footnotetext{
${ }^{1}$ The author of this paper was unable to attend the Colloquium - eds.
} 


$$
\begin{aligned}
& \rho_{c}=\text { the radius of curvature of the apparent orbit at the point } \\
& \text { corresponding to } t_{0} .
\end{aligned}
$$

To determine the AMP-orbit we also used a value of the radial velocity of the component $\mathrm{B}$ relative to $\mathrm{A}, \delta V_{\mathrm{r}}=-3.2 \pm 0.2 \mathrm{~km} \mathrm{~s}^{-1}$ (Tokovinin 1988), and a value of the trigonometrical parallax $\pi_{t}=0^{\prime \prime} .094 \pm 0^{\prime \prime} .004$ (Woolley et al. 1970). The results are presented in Tables 1 and 2 in the line $P$.

TABLE 1. The apparent motion parameters at the moment $t_{0}$

\begin{tabular}{|ccrrcrc|}
\hline & \multicolumn{1}{c}{$t_{0}$} & \multicolumn{1}{c}{$\rho$} & \multicolumn{1}{c}{$\theta$} & \multicolumn{1}{c}{$\mu$} & \multicolumn{1}{c}{$\Psi$} & \multicolumn{1}{c}{$\rho_{c}$} \\
\hline $\mathrm{P}$ & 1980.0 & $5^{\prime \prime} .964$ & $173^{\circ} .45$ & $0^{\prime \prime} .0460$ & $250^{\circ} .82$ & $3^{\prime \prime} .3$ \\
& & \pm 0.002 & 0.01 & 0.0002 & 0.33 & 0.5 \\
$\mathrm{~W}+\mathrm{P}$ & 1974.0 & 5.899 & 170.84 & 0.0465 & 246.11 & 3.4 \\
& & \pm 0.001 & 0.01 & 0.0002 & 0.20 & 0.2 \\
\hline
\end{tabular}

TABLE 2. The elements of the orbits

\begin{tabular}{|crrrrrrrr|}
\hline & $\alpha$ & $\begin{array}{c}\mathrm{P} \\
(\mathrm{yr})\end{array}$ & $e$ & $\omega$ & $i$ & $\Omega$ & $\begin{array}{c}\mathrm{T}_{P} \\
(\mathrm{yr})\end{array}$ & $\begin{array}{c}\mathcal{M}_{A+B} \\
\left(\mathcal{M}_{0}\right)\end{array}$ \\
\hline $\mathrm{P}$ & $13^{\prime \prime} .3$ & 1992 & 0.55 & $164^{\circ}$ & $55^{\circ}$ & $357^{\circ}$ & 1967 & 0.72 \\
& \pm 5.5 & 817 & 0.16 & 21 & 3 & 7 & 778 & 0.09 \\
$\mathrm{~W}+\mathrm{P}$ & 13.5 & 2018 & 0.56 & 165 & 56 & 357 & 1967 & 0.72 \\
& \pm 5.6 & 744 & 0.14 & 15 & 3 & 7 & 404 & 0.09 \\
\hline
\end{tabular}

For comparison we used also the set of observations obtained with the 26inch refractor at the U.S. Naval Observatory from 1958 through 1975.

Unfortunately, this set was not sufficient for calculating the radius of curvature $\rho_{c}$, and we could not determine the independent orbit. Therefore we determined the orbit on the basis of the united set of Washington and Pulkovo observations. The new arc consists of $1958-1989$ years observations $\left(14^{\circ}\right.$ length $)$.

The analysis of the common portion of the set including $1968-1975$ years yielded similar waves in the motions corresponding to the Pulkovo and Washington observations and the small systematical differences which were taken into consideration: (W-P) $-\rho=+0^{\prime \prime} .001,(\mathrm{~W}-\mathrm{P})_{\theta}=+0^{\circ} .012$.

The results are presented in Tables 1 and 2 in the lines $(\mathrm{W}+\mathrm{P})$. The errors in the orbital elements depend on the initial data errors. The orbital period is determined uncertainly, but the values of all elements are close values. The orbits satisfy the ancient observations.

The new value of $\mathcal{M}_{A+B}$ is in agreement with the "mass-luminosity" relation more or less. We assume that the reason for a difference between the new orbits and the first one is in the measuring technique. The plates of the different years were measured by different persons before, and we did not pay special attention to this question. The different personal errors of the measurers introduced the essential systematical errors into the value of the apparent velocity, and we got a wrong effect. 

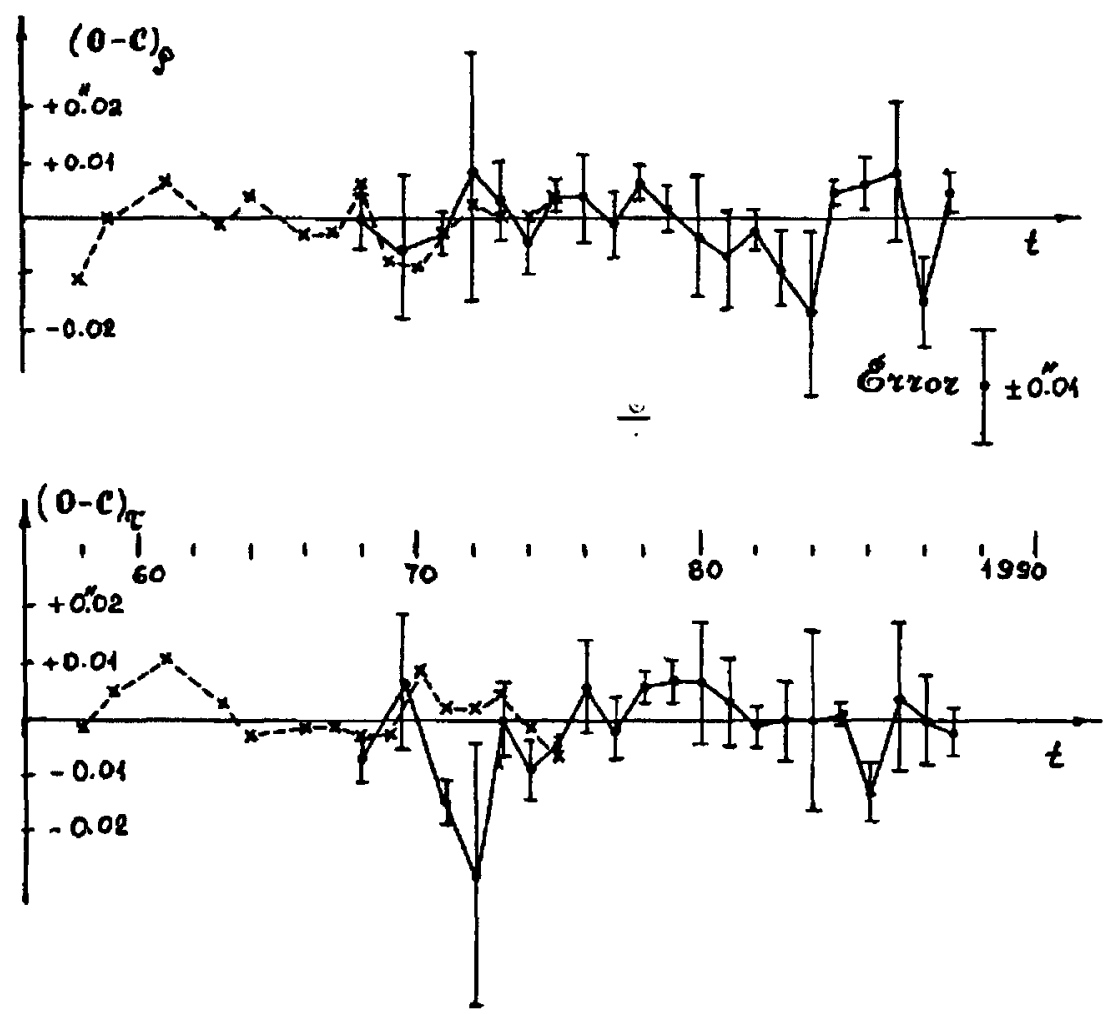

FIGURE 1. Deviations in the observations relative to the $(W+P)$ orbit. Dots with error bars refer to Pulkova observations, $x$ 's to USNO observations.

The most important result of this investigation is in the discovery of similar waves in both independent sets of observations (see Figure 1). These waves have a small amplitude about $0^{\prime \prime} .003-0^{\prime \prime} .005$ (it is less than an effect of accidental errors) and a period about 3.5 years, but they are repeated with the same phases in the Washington and Pulkovo observations. Consequently, they cannot be accidental deviations, and we can suppose the existence of an invisible satellite with a mass about $0.01 \mathcal{M}_{\odot}$.

\section{REFERENCES}

Kisselev A.A. \& Kiyaeva O.V. 1988, Ap\&SS, 142, 181

Güntzel-Lingner U. 1955, Mitt. Astrophys. Obs. Potsdam, 42, 183

Tokovinin A.A. 1988, Astrophys. USSR, 28, 2, 297

Woolley, R., Epps, E.A., Penston, M.J., \& Pocock, S.B. 1970, Catalogue of Stars within 25 parsec of the Sun, Royal Obs. Annal 5 